\title{
Canadian Kidney Cancer Forum 2008
}

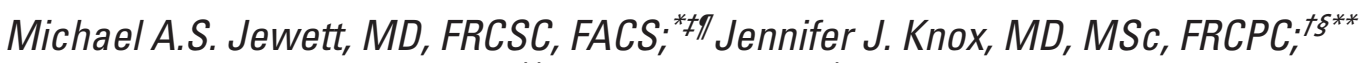 \\ Christian Kollmansberger, MD; ${ }^{\text {t十 }}$ Joan Basiuk, $\mathrm{RN}^{\dagger}$
}

See related article on page 175

I n Canada, at least 4500 people are diagnosed with kidney cancer, and 1 in 3 die of the disease. It is the most lethal genitourinary cancer, and its incidence is increasing. Unlike most human cancers, there is considerable knowledge about specific genetic changes in these cancers. Major advances in treatment are being made owing to new systemic targeted therapies as well as less morbid technologies for localized cancers. The first Canadian Kidney Cancer Forum was held Jan. 31-Feb. 2, 2008, in Mont Tremblant, Quebec, to review these developments and identify opportunities for Canadians. The primary meeting objectives were achieved and included:

1. Achievement of consensus among expert professionals and patients on the management of patients with localized, locally advanced and metastatic renal cell carcinoma, as well as the in the areas of pathology and imaging. Over 3 days, a series of sessions were held to address the different stages of disease and issues. In this issue, we present the consensus statements. Key references in each area were provided by experts and were graded using a modified version of the Oxford Levels of Evidence. During the conference, experts in each area presented, followed by an opportunity for questions and discussion. At the end of the forum, overviews were presented, and participants voted on draft statements developed during the sessions.

2. Stimulation of clinical research in kidney cancer including opportunities for national and international collaboration. To achieve this, the formation of a research network was proposed with the suggested name, Kidney Cancer Research Network Canada.

3. Definition of educational needs to improve kidney cancer care in Canada. This included discussing a national kidney cancer database that will be developed.

4. Initiating the development of a cancer control strategy for kidney cancer to ensure all Canadians have equal access to care to attempt to develop a national approach to the management of this cancer.

The Forum was endorsed by the Canadian Urological Association, the Canadian Association of Medical Oncologists and the Canadian Association of Radiation Oncologists, and was supported by grants from the Canadian Institutes of Health Research, the National Cancer Institute of Canada, the Canadian Cancer Society, the Canadian Partnership Against Cancer, several provincial cancer agencies (Cancer Care Ontario, Cancer Care Manitoba, Cancer Care Nova Scotia, Alberta Cancer Foundation) and the Princess Margaret Hospital Foundation, and industry. These groups had been identified by the steering committee as ones that had demonstrated expertise, interest or experience in clinical or research aspects of kidney cancer. They were joined by a small number of invited participants from Europe and the United States to bring an international perspective to the process. Dr. George Browman, representing the Canadian Partnership Against Cancer, contributed expertise in the development and use of guidelines for cancer care.

Overall, the participants believe that the forum will have a significant impact on the care of Canadians afflicted with kidney cancer. Given the enormous success of this first meeting, planning for a second meeting in 2009 has commenced.

From the Departments of *Surgery, Division of Urology, and †Medicine, University Health Network, Toronto, Ont., the Departments of $\ddagger$ Surgical Oncology and §Medical Oncology, Princess Margaret Hospital, Toronto, Ont., the Departments of $\uparrow$ Surgery (Urology) and ${ }^{*}$ Medicine, University of Toronto, Toronto, Ont., and the ††Division of Medical Oncology University of British Columbia, Vancouver, BC

Competing interests: None declared.

Correspondence: Dr. Michael A.S. Jewett, Division of Urology, University of Toronto, 610 University Ave., 3-124, Toronto ON M5G 2C4; m.jewett@utoronto.ca 